\title{
Literature Review of Supply Chain Finance Based on Blockchain Perspective
}

\author{
Zhongmin Liu \\ School of Economics \& Management, Shanghai Maritime University, Shanghai, China \\ Email: 47350905@qq.com
}

How to cite this paper: Liu, Z. M. (2021). Literature Review of Supply Chain Finance Based on Blockchain Perspective. Open Journal of Business and Management, 9, 419-429.

https://doi.org/10.4236/ojbm.2021.91022

Received: May 11, 2020

Accepted: January 26, 2021

Published: January 29, 2021

Copyright (c) 2021 by author(s) and Scientific Research Publishing Inc. This work is licensed under the Creative Commons Attribution International License (CC BY 4.0).

http://creativecommons.org/licenses/by/4.0/

(c) (i) Open Access

\begin{abstract}
Blockchain technology has the characteristics of decentralization and immutability. Since it appears, blockchain technology has received widespread attention and is considered to be the most revolutionary technology with the most promising development after cloud computing, Internet accelerated speed, and big data. Therefore, a wave of application of blockchain technology has set off around the world today. Supply chain finance is based on multi-party, relatively closed environment, and requires controllable and trusted data as supporting factors. Naturally, it has great potential room for combining with blockchain technology. The application of blockchain technology in the field of supply chain finance can provide new solutions to the problems in the development of supply chain finance. Therefore, the research on supply chain finance based on blockchain has become a new research hotspot. This article reviews the current literature on the application of blockchain to supply chain finance. Numerous studies have shown that the application of blockchain technology will open a new door for the development of supply chain finance and promote the vigorous and healthy development of supply chain finance.
\end{abstract}

\section{Keywords}

Blockchain, Supply Chain Finance, Literature Review

\section{The Creation and Evolution of Supply Chain Finance}

With the continuous deepening of socialized production methods, market competition has shifted from competition between single customers to competition between supply chains. At the same time, as the existence of fund accounts has become a mainstream feature of transactions, it is difficult for suppliers in the middle and upper reaches of the supply chain to obtain bank funding support 
through "traditional" credit methods. Supply chain finance is a financial innovation business of financial institutions such as commercial banks. The biggest difference between traditional credit businesses is that supply chain finance uses the credit capabilities of core companies in the supply chain and third-party logistics companies to alleviate the asymmetry of information between financial institutions such as commercial banks and small and medium enterprises, and solve the problem of lack of mortgage and guarantee resources for small and medium enterprises.

The development of western supply chain finance can be roughly divided into three stages. The development of western supply chain finance can be roughly divided into three stages. For the first stage, the business of supply chain finance is very simple, mainly for the loan business of inventory pledge. In the second stage, the supply chain finance business began to be rich, and factoring businesses such as off-take receivables began to emerge. However, the supply chain finance business at this stage is still "mainly pledged inventory, supplemented by accounts receivable". In the third stage, the business of supply chain finance began to flourish, and financing products such as prepayment financing, settlement and insurance appeared. This is due to the high concentration of the logistics industry and the development of supply chain theory. In the late 1980s, major international logistics began to gradually focus on a few logistics companies, such as FedEx, UPS, and German Rail Logistics. At this stage, the development of foreign supply chain finance began to form a "logistics-based, financial supplementary" operating philosophy, and supply chain finance has developed rapidly due to the deep participation of logistics companies.

The development of Chinese supply chain finance depends on the rapid development of manufacturing in the 30 years of reform and opening up. At this stage, the development of China's supply chain finance presents several characteristics.

Firstly, regional development of supply chain finance is uneven. coastal areas where the export-oriented economy is relatively obvious, the development of supply chain finance is relatively advanced, while inland supply chain finance is still in its infancy. Secondly, Chinese supply chain finance is still facing legal risks. There is still a certain legal vacuum in the pledge of current assets such as inventory goods. The status quo of Chinese banking division operation has resulted in a variety of principal-agent relationships in the supply chain financial business, and the backwardness of China's social credit system construction has further caused the operation risk of supply chain financial business.

Currently, the research on supply chain finance is more about analyzing financing models, risk and management pricing, cooperation and games under traditional technology conditions.

However, the application of innovative technologies to the field of supply chain finance to address the needs of supply chain finance development has not been studied in depth. This article summarizes the literature on the application 
of blockchain technology to the development and innovation of supply chain finance, and gives a literature review of this research.

\section{Overview of Blockchain Technology Application}

Many scholars started from the technical advantages of blockchain and the business characteristics of supply chain finance, and explained in a macro perspective that applying blockchain technology to the field of supply chain finance can effectively improve the service capabilities and efficiency of supply chain finance. This reveals the great potential and practical significance of blockchain technology applied to supply chain finance. (Kelly, 2019) examines the essential misconceptions about blockchain technology and addresses the means of identifying the truth behind a popular and hotly debated topic. One of the biggest obstacles to blockchain technology, other than it still being in its adolescent phase, is the number of misconceptions about what the technology is, what it does and what it can do.

The pain points in the supply chain finance field and the advantages of blockchain technology in the financial field (Huang \& Gan, 2018), and proposed the use of blockchain technology to reconstruct the supply chain financial service model, which can well solve the pain points in the supply chain finance field. (Zhao et al., 2020) analyze how blockchain technology can empower the logistics industry, combine blockchain technology with logistics, form a new mode of "blockchain + intelligent logistics", promote the application of blockchain technology in the logistics industry, explore more and deeper application scenarios, promote the reform of the logistics industry, and help the logistics industry move towards a higher level effective and more coordinated development.

The reconstruction of the supply chain financial model by the blockchain firstly requires the establishment of an alliance chain based on supply chain finance. The alliance chain mainly includes core enterprises, various financial institutions, financial intermediaries, suppliers and sellers at all levels, and blockchain service platforms. Blockchain technology (Zhang, 2018), while ensuring the security of financial data in the supply chain, has promoted the improvement of credit efficiency and quality of core enterprises, and reduced the risk of supply chain financial business of financial institutions. At the same time, relying on blockchain technology to build a comprehensive information platform covering all nodes in the industry, not only can promote the trust relationship between enterprises in the industry chain, but also can realize the unified management of capital flow, information flow, business flow and logistics, thereby improving the production efficiency and capital utilization efficiency of the entire industrial chain, and promoting the coordinated development of the entire industry. The blockchain itself ( $\mathrm{Wu}, 2019)$ has the characteristics of transparent and non-tamperable information, so its application in supply chain finance helps information exchange and interoperability, and makes trust deli- 
very better in supply chain finance (Chu \& Gao, 2018). Blockchain promotes the development of supply chain finance from three aspects: the realization of information symmetry among participating parties, the realization of core enterprise credit transfer, the realization of supply chain financial process visibility, risk controllability, and full service coverage. At the same time, the application of blockchain in the supply chain financial business can be divided into three categories: financial institutions dominate, trading platform enterprises dominate, and third-party platform enterprises dominate. The theoretical foundation of introducing blockchain technology into supply chain finance with the help of micro-financial innovation model (Wang, 2018b), and constructed an ecological model of supply chain financial industry organization, from the vertical dimension based on this, an integrated solution for blockchain supply chain finance and horizontal optimization of main financing models are proposed (Zhou \& Li, 2016). There are many bottlenecks in the promotion of supply chain such as the limitation of credit objects, the limitation of technology integration, and the visibility of the entire transaction process. The application of blockchain technology is helpful for the promotion of supply chain financial services, and is the best solution to help banks break through the above bottlenecks (Zhu et al., 2018). The parties in the supply chain jointly build an alliance chain, and all parties can share a transparent and reliable information platform and traceability processes, and can also conduct supply chain finance and precision marketing based on blockchain data. Blockchain technology (Wang, 2018a) will make small and medium-sized enterprises in the industry chain an important node in the allocation of financial resources, and will also promote the improvement of the efficiency of the existing financial sector, construct a new model for the development of a shared win-win supply chain finance. The application of blockchain (Li, 2019) from three aspects of capital flow, logistics and supply chain financial information flow, and how its operation model should be improved and the final effect, and proposed corresponding solutions. Blockchains could revolutionize the underlying technology of the payment clearing and credit information systems in banks (Guo \& Liang, 2016), thus upgrading and transforming them. Blockchain applications also promote the formation of "multi-center, weakly intermediated" scenarios, which will enhance the efficiency of the banking industry.

At the same time, many scholars have discussed the strategies of blockchain technology applied to the field of supply chain finance in more detail from the micro perspective of the development of supply chain finance.

From the perspective of supply chain financial innovation (Zhao, 2018), constructed a supply chain financial business development model from the perspective of blockchain technology characteristics, and analyzed the development of supply chain financial innovation from the aspects of function realization, core technology, and business modules. (Zheng, 2019) proposed a network supply chain financial innovation development path based on blockchain technology, which specifically includes three aspects: 1) Relying on blockchain thinking to 
build a cloud platform and constructing an ecosystem; 2) Blockchain technology-based business Implementation ideas; 3) Upgrade the management tools adapted to the application of blockchain technology in network supply chain finance. (Lin, 2019) took the characteristics of the supply chain financial model as the starting point, integrated the blockchain technology into the supply chain financial industry, and established the "supply chain finance based on the core enterprise to establish a blockchain framework" model and the Internet Financial companies establish a supply chain finance model of the blockchain framework based on the analysis of the advantages of two "blockchain plus supply chain finance" models. (Zhang, 2019) analyzed the supply chain financial service model and the blockchain incentive mechanism from the perspective of the game, and aimed at the pain points of the supply chain finance, gave a specific description of the blockchain-driven supply chain finance Innovative development ideas and countermeasures. It is possible to build an open and shared supply chain financial credit system by embedding blockchain technology (Liu \& Li, 2019). The system can be built from five aspects: 1) Construction of an open and shared credit framework. 2) Establishing a credit reporting platform combining officials and citizens. 3) Improving the sense of responsibility of stakeholders. 4) Establish a full life cycle monitoring mechanism. 5) Improve the shared list of red and black lists. (Yeh \& Kashef, 2020) call for innovative and advanced research on blockchain and recommendation systems. We aim at building a secure and trust-based system using the advantages of blockchainsupported secure multiparty computation by adding smart contracts with the main blockchain protocol.

From the perspective of supply chain financing, (Zhang et al., 2019) analyzed the feasibility of using the alliance chain to solve the problem of multi-party trust relationship in supply chain finance, to solve the problem of supply chain trust transfer and split circulation, and to improve the efficiency of supply chain financial business. With combination of practice, it described in detail all aspects of contract signing, confirmation of creditor's rights, accorporating financing, transformation of creditor's rights, and how to apply the characteristics of the alliance chain to solve practical problems, and summarized and prospected the supply chain finance application based on the blockchain. (Tang \& Zhuang, 2019) used the newsboy model to compare and analyze the blockchain debt transfer platform model and the traditional supply chain financing model from the perspective of the differences in the benefits of various entities in the supply chain and discuss the advantages of the blockchain debt-to-platform model in reducing costs, improving returns, and promoting turnover. Combing with the characteristics, architecture and core technology of blockchain, and puts forward corresponding solutions (Zhu \& Wang, 2020), blockchain technology removes many constraints in various system applications and provides technical possibilities for the realization of many ideas. This paper summarizes that blockchain technology has a good application prospect in the field of logistics supply chain, 
which can deal with the long-standing problems in the field of logistics and is expected to bring changes to the logistics industry.

Studies have shown that a blockchain debt-to-debt platform can increase supplier output, increase the revenue of each member of the supply chain, and maximize the financing benefits of receivables. (Ju \& Tang, 2020) introduce the application of blockchain technology in supply chain finance 4.0 mode, and puts forward relevant policy suggestions based on the existing development situation.

From the perspective of supply chain financial risk control, blockchain technology (Chen, 2019) can optimize the supply chain financial risk control in terms of expanding the scope of credit granting, ensuring the authenticity of information, and reducing operational risks. (Gao, Zhou et al., 2018) discussed SCF pledge risk control based on blockchain technology, which is known for information transparency and tamper proof, and proposed a real-time Stare in Market (RTSM) strategy to alleviate the risk pressure brought by chattel pledge. Replenishment is required once total pledges fall below the safe level. The results of numerical experiment show that block chain technology not only plays an important role on the risk control of network supply chain, but also breaks down the "barrier", expands the risk control scope of supply chain to two or three levels of suppliers and distributors, and expands the business scope of network supply chain. It is proved that the RTSM strategy can not only effectively avoid the bank's pledge risk, but also make the bank gain more profits. It is formally defined by the reason about the security of our protocols, we are the first to formalize the blockchain model of cryptography. The formal modeling is of independent interest. We advocate the community to adopt such a formal model when designing applications atop decentralized blockchains.

The Blockchain Technology (BCT) for Supply Chain Finance (SCF) programs allows businesses to come together in partnerships and accelerate cash flows throughout the supply chain (Hofmann et al., 2018). Plus, Blockchain and distributed ledgers technologies could deliver substantial benefits for all parties involved in SCF transactions, promising to expedite the processes and lower the overall costs of financing programs, aim to reduce complexity and make data sharing more secure, accurate and efficient.

In addition, some scholars have studied the supply chain finance system based on blockchain, and proposed the specific application framework and operation mode of the combination of blockchain technology and supply chain finance.

The development status of supply chain finance and "blockchain plus supply chain finance" platform (Long et al., 2019), and based on the problems of the platform, broke down the supply chain finance platform based on blockchain technology into four modules: authority management, credit management, contract management and tracing. (Panuparb, 2019) proposed a new cost-benefit model and the operation process of traditional and blockchain-based supply chain finance solutions, taking the supply chain in Thailand as a research example, to evaluate and predict the net value of the implementation of blockchain 
technology by relevant parties. It is proposed that the total net income of the parties involved in the supply chain financing arrangement increases correspondingly due to the improved invoice processing efficiency. If suppliers pay less for the platform than to unlock the working capital, they will benefit from blockchain-based supply chain financing, while buyers will not get benefit (Omran et al., 2017). The conceptual framework of a blockchain-driven supply chain finance (SCF) solution. The reference framework is designed to facilitate coordination between buyers and suppliers and eliminate inefficiencies in the implementation of discrete supply chain finance instruments, such as reverse factoring and dynamic discounting. In addition, the author also introduces the value drivers of blockchain technology (BCT) to illustrate its unique characteristics in the application of SCF. A single cycle SCF system (Zhu \& Dong, 2018) based on blockchain platform. Suppose the supplier faces a funding constraint and must borrow through the online blockchain platform. The blockchain platform avoids risks by setting service rates, guarantee fees and loan fees. The study found that the optimal strategy for participants in the SCF system is Stackelberg. For the supply chain finance system with capital supply constraints, the interest rate of the blockchain platform service provided to the supplier is related to the default rate of the supplier and the loan amount of the blockchain platform service to the supplier. The analysis shows that it is very important for suppliers to consider the financial decisions of the online blockchain platform (such as service rate) when making operational decisions. Based on the analysis of the existing supply chain technology bottleneck model, (Ju \& Tang, 2020) put forward some suggestions. (Zhao \& Huchzermeier, 2020) explain how the operations-finance interface jointly optimizes material and financial flows under intricate risk exposures. (Wang \& Chen, 2020) suggested that in accordance with the four-dimensional development mode of "multi-party credit co-construction + regulatory technology upgrading + standard platform system + digital transformation of circular", multi-party exploration and co-construction of multi-layer supply chain financial system based on blockchain technology should be encouraged, scientific and technological supervision should be strengthened, the "multi-party + regulatory" pattern of blockchain should be realized, a standardized supply chain financial service platform should be built, and circular ecology should be built to promote regional development blockchain technology helps the effective implementation of Inclusive Finance.

Blockchain technology can realize the online interconnection of the whole chain of the supplychain (Yi et al., 2020), make the communication and transaction behavior of all parties in the supplychain finance completely networked, make the information transmission between each other more efficient and quick, and the funds of enterprise financing get to the account more quickly, and the financial platform constructed using blockchain technology can best realize the convenience, real-time and accuracy of the process in transaction, and reduce the possibility of dishonest behaviors that occur in transaction, improve the 
convenience of trade financing, which constructs a higher-quality, information-symmetrical transaction mode for the society.

Wang, Yan, \& Fu (2020) put forward the optimal analysis of agricultural product supply chain model construction and related node risk management under blockchain technology. The main cause of various risk factors is caused by the imperfect coordination and lack of information sharing between the nodes in the agricultural product supply chain; taking this as a starting point, combined with the technical characteristics of decentralization, information security and transparency in blockchain technology, analyzed the fit and technical application of blockchain technology in the risk management of agricultural product supply chain, establishing a risk management structure of agricultural product supply chain under blockchain technology, and analyzes the process and elements of risk management of agricultural product supply chain under blockchain technology. (Gao, 2021) building a new rural financial information sharing platform, building a smart contract-based rural financial asset trading platform, advancing blockchain technology to integrate finance innovative products, coordinated supervisions and boxes and improving the supervision system of rural financial institutions, and constructing a blockchain agricultural insurance application mechanism. It provided a new way of thinking to alleviate the urgency of rural financial reform under the background of agricultural supply side.

(Cao, 2021) explores the impact of blockchain smart contract on banking business, and explores a new financial business model based on blockchain technology and smart contract.

Besides, it is helpful to improve the integration of blockchain technology and Internet Finance and give full play to the positive effect of Internet Finance by formulating risk prevention strategies of Internet finance from four aspects: in-creasing technology research and development, improving regulatory mechanism, standardizing industry standards and training special talents (Zhang \& Wang, 2021).

In response to the credit risks, legal risks, peratioanl risks, and business risks faced by the development of supply chain finance business (Xue, 2021), using blockchain technology to actively carry out a large number of practical explorations, achieving iterative product system upgrades, financing model innovation and long-tail customers expansion, effectively reducing of commercial banks, accelerate the improvement of technical legal frameworks, build a blockchain technology application framework system, innovate supply chain financial models, and promote the sustainable development of commercial banks and supply chain financial services.

(Sun et al., 2021) introduce blockchain technology and issue digital bills through the characteristics of decentralization and distrust of blockchain technology to assist supply chain finance in many ways, help SMEs solve the problem of financing difficulties, and provide a low-cost, low-risk, high transparency and more efficient financing environment for SMEs. 


\section{Summary and Outlook}

The combination of blockchain and supply chain finance plays an important role in many industries. It not only improves the efficiency of operation process, but also credit access is also guaranteed highly qualified. In terms of the development of internet industry, it shows more and more important in the supply chain field in application of blockchain.

The application of blockchain technology in supply chain finance has brought new opportunities and challenges to the development of supply chain finance. The application of blockchain technology effectively guarantees the security and reliability of information transmission, expands the credit scope of supply chain enterprises, reduces the financing cost of small and medium-sized enterprises, and enhances the risk control ability of the banking industry.

In general, the blockchain technology is applied to supply chain finance, the omnidirectional dynamic supervision and security mechanism formed, the "four streams in one" visual management, and the digitization of all parties' capital transactions can form a comprehensive risk prevention network, which greatly improves the security of supply chain finance. Although blockchain has great potential in the field of supply chain finance, there are still some issues worth further study and discussion:

1) to what extent can the application of blockchain improve the efficiency of supply chain finance and how to quantify these improvements.

2) how to transform the business model of supply chain finance, and give specific application strategies and model framework of blockchain, so as to make it better adapt to the development needs of supply chain finance by combining blockchain technology.

3) the regulation of supply chain finance under the blockchain will be a new topic.

It is believed that with the deepening of blockchain research and application, blockchain will greatly change the form of supply chain finance and improve the efficiency of supply chain finance.

4) facing the huge market and customers, how to use the blockchain technology to guarantee the credit application and safety in manufacturing industry and logistics companies.

\section{Conflicts of Interest}

The author declares no conflicts of interest regarding the publication of this paper.

\section{References}

Cao, L. (2021). Blockchain Smart Contract and Banking Business Development. FinTech Time, 1, 40-43.

Chen, J. M. (2019). Optimization of Supply Chain Financial Risk Control by Blockchain Technology. China Economist, 1, 29-31. 
Chu, X. J., \& Gao, B. (2018). Research on the Financial Innovation of Supply Chain Driven by Block Chain. Research on Financial Development.

Gao, B. H., Zhou, Q., Li, S. G. et al. (2018). A Real Time Stare in Market Strategy for Supply Chain Financing Pledge Risk Management. 2018 IEEE International Conference on Industrial Engineering and Engineering Management (IEEM), Bangkok, 16-19 December 2018. https://doi.org/10.1109/IEEM.2018.8607805

Gao, C. (2021). Solving Risk Problems in Rural Finance Based on Blockchain Technology. China Forest Economics, 1, 117-120.

Guo, Y., \& Liang, C. (2016). Blockchain Application and Outlook in the Banking Industry. Financial Innovation, 2, Article No. 24. https://doi.org/10.1186/s40854-016-0034-9

Hofmann, Erik, \& Streew et al. (2018). Supply Chain Finance and Blockchain Technology: The Case of Reverse Securitisation. Softcover Springer Buiefs.

Huang, X. J., \& Gan, T. (2018). Analysis on the Application of Block Chain in the Field of Supply Chain Finance. Journal of Changchun Finance College, 4, 62-66.

Ju, J., \& Tang, C.-H. (2020). Research on Supply Chain Finance 4.0 Mode Based on Blockchain Technology. Financial Perspectives Journal, 3, 57-62.

Kelly, P. (2019). Discerning between Hype and Reality: Common Misconceptions about Blockchain. Journal of Digital Banking.

Li, B. (2019). The Transformation of Supply Chain Financial Mode by Block Chain Technology. Management \& Technology of SME, 2, 70-71.

Lin, N. (2019). Research on Supply Chain Financial Model Innovation Based on Blockchain Technology. New Finance.

Liu, S L., \& Li, H. M. (2019). On the Optimization of Supply Chain Financial Credit Reporting System by Embedding Blockchain Technology. Credit Reference, 8, 16-20.

Long, Y. N., Zhang, J., \& Ai, R. (2019). Research on the Optimization of Supply Chain Financial System Based on Blockchain Technology. Southwest Finance, 1, 72-79.

Omran, Y., Henke, M., Heines, R. et al. (2017). Blockchain-Driven Supply Chain Finance: Towards a Conceptual Framework from a Buyer Perspective.

Panuparb, P. (2019). Cost-Benefit Analysis of a Blockchain-Based Supply Chain Finance Solution. Cambridge: Massachusetts Institute of Technology.

Sun, H., Zhou, Z. Y., \& Fu, T. S. (2021). Research on Financing of Small and Medium Sized Enterprises under the Background of "Blockchain + Supply Chain Finance". Modern Business, 3, 48-50.

Tang, D., \& Zhuang, X. T. (2019). Research on Decision-Making of Supply Chain Finance Based on Blockchain Accounts Receivables Transfer Platform. System Engineering, 37, $58-66$.

Wang, H. (2018a). Blockchain Technology and New Mode of Supply Chain Finance. China CIO News, $133+137$.

Wang, K. Y., Yan, X. X., \& Fu, K. Y. (2020). Research on Risk Management of Agricultural Products Supply Chain Based on Blockchain Technology. Open Journal of Business and Management, 8, 2493-2503. https://doi.org/10.4236/ojbm.2020.86155

Wang, X., \& Chen, L. Y. (2020). Advantages, Value and Construction of Multi-Layer Supply Chain Finance System Based on Blockchain Technology: From the Microview of Credit Loss-free Transmission. South China Finance, 60-68.

Wang, Y. (2018b). The Application Study of Block Chain Technology in Supply Chain Finance. Beijing: University of International Business and Economics.

Wu, Q. T. (2019). Application of Blockchain Technology in Supply Chain Finance. Mod- 
ern Marketing (Information Version).

Xue, Y. (2021). The Innocatice Path of Blockchain Technology to Drive the Commercial Banks to Develop Supply Chain Financial Services. Southwest Finance, 2, 38-48.

Yeh, T.-Y., \& Kashef, R. (2020). Trust-Based Collaborative Filtering Recommendation Systems on the Blockchain. Advances in Internet of Things.

Yi, Z. L., Zhang, H. F., \& Gong, Z. H. (2020). Innovation and Discussion on the Application of Blockchain Technology under the Supply Chain Financial Mode. Management \& Technology of SME, 4, 44-47.

Zhang, C. H., \& Wang, X. (2021). Risk Prevention of Internet Finance under Blockchain Technology. China Economic \& Trade Herald.

Zhang, G. H., Zhao, K. Q., \& Hou, W. B. (2019). Research on Supply Chain Financing Innovation Based on Blockchain Technology. Information Technology and Network Security, 94-95.

Zhang, L. (2019). Research on Financial Innovation of Supply Chain Driven by Blockchain from the Perspective of Game Theory. On Economic Problems, 4, 48-54.

Zhang, Q. B. (2018). Analysis of the Optimization Effect of Blockchain Technology on Supply Chain Finance. Hebei Enterprises, 8, 119-120.

Zhao, L. (2018). Research on Supply Chain Finance Innovation and Development Based on Blockchain Technology. Logistics Engineering and Management, 40, 74-75.

Zhao, L. M., \& Huchzermeier, A. (2020). On the Framework and Mode Categorization of International Supply Chain Finance. New Economy, 73-85.

Zhao, T., Sun, X. Y., Qiu, P. W., \& Li, D. G. (2020). Research on Optimization of Intelligent Logistics Mode Based on Blockchain. Cyberspace Security, 11, 78-83.

Zheng, J. Y. (2019). On the Optimization Path of Financial Innovation of Network Supply Chain Based on Blockchain Technology. Journal of Shanxi Finance and Economics University, 41, 18-20.

Zhou, L. Q., \& Li, Z. H. (2016). Application of Blockchain in Supply Chain Finance. China CIO News, 49-51.

Zhu, C. Y., \& Dong, A. Q. (2018). The Coordination Mechanism of Supply Chain Finance Based on Block Chain. IOP Conference Series: Earth and Environmental Science (Vol. 189). IOP Publishing. https://doi.org/10.1088/1755-1315/189/6/062019

Zhu, X. X., He, Q. S., \& Guo, S. Q. (2018). On the Role of Blockchain Technology in Supply Chain Finance. China Business and Market, 32, 111-119.

Zhu, X., \& Wang, Y. (2020). Research on the Application of Blockchain Technology in the Field of Supply Chain. 2020 International Conference on Advanced Education, Management and Information Technology (AEMIT2020) (pp. 154-157), Paris: Atlantis Press. https://doi.org/10.2991/assehr.k.200727.034 